\title{
Malformaciones cardíacas congénitas en el síndrome alcohólico fetal
}

\author{
Dra. María Mena R. 1; Dr. Víctor Pacheco C.2; Dr. Alexis Lama T.2; \\ Dr. Carlos Otero V.2; Dr. Jaime Tapia Z,3; Dr. Richard Sthurman R.4
}

\section{Congenital heart disease in children with severe fetal alcohol syndrome}

Among 130 children with severe fetal alcohol syndtomes (FAS), seen at Concepción-Chile, 17 (13\%) showed evidence of congenital hear disease (CHD). One third of the malfomations corresponded to septal defects, and one fourth to Fallot's tetralogy. Five patients died: three babies at the newborn period; two infarts after surgical interventions. The remaining 12 children's curyent cardiac functional capacity is fairly we!l. The frecuent association of CHD and FAS is emphasized. The final outcome will depend on the severity of the underly ing congenital heart disease and the mental and social disturbances related to alcoholism as wel. Prevention is the mainstay of management in FAS.

(Key words: Fetal alcohol syndrome, heart malformations, congenital heart disease).

Además de las características clínicas que se describen en el sindrome alcohólico fetal $(\mathrm{SAF})^{1}$, se pueden observar también malformaciones de diferentes órganos: renales ${ }^{2,3}$, oculares $^{4}$, hepáticas $5^{5,6}$, del tubo neural ${ }^{7}$, entre otros, etc. La asociación del síndrome con cardiopatias congénitas ha sido comunicada con frecuencias de $29 \%$ y $50 \%$ en distintas series extranjeras $^{8}, 9,10,11,12$. El objetivo principal de esta investigación fue estudiar la asociación de cardiopatías congénitas con SAF con fines educativos y

1. Servicio de Pediatría, Unid de Genética. Hosp. G. Gran B., Concepción.

2. Unidad de Cardjologia. Hosp. Gmo. Grant B., Concepción.

3. Becado Depto, Pediatría, Fac. de Medicina, Universidad de Concepción.

4. Anatomía Patológica, Facultad de Medicina, Universidad de Concepción. preventivos; identificar las malformaciones car diacas predominantes, observar el pronóstico y evolución de ellas e intentar relácionar la canti. dad y el modo de ingerir alcohol durąnte el embarazo con la existencia de anomalías cardiacas en los niños.

\section{MATERIAL Y METODO}

En la unidad de genética del hospital Guillemo Grant Benayente de Concepción, entre los años 1980 y 1985, fueron identificados 130 casos de SAF severos. Los pacientes con síntomas y signos de trastorno cardiovasculares, fueron estudiados en la unidad de cardiología. Se indagaron y registraton en cada caso antecedentes prenatales de ingestión de drogas, infeccio. nes virales y uso de medicamentos antes y después del embatazo, con la finalidad de descartar otras causas de cardiopatías congénitas. El díagnóstico de las malformaciones cardíacas fue documentado por los signos y exámenes complementarios, en especial ecocarđiografía M y cuando fue requerido, estudios hemodinámicos y cineangiocardiográficos. Algunos pacientes fueron sometidos a intervención quirúrgica $y$ se realizaron necropsias en los que fallecieron, cuando ello fue posible. 


\section{RESULTADOS}

Se encontraron 17 pacientes con malformaciones congénitas del corazón (MCC) entre los $130 \mathrm{SAF}(13 \%)$. No siempre fue posible obtener información directa sobre el consumo diario de alcohol de las madres durante el embarazo, pero en general fue de alrededor de 1 L de vino al día en promedio: la mayoría eran bebedoras excesivas, 3 habían fallecido de cirrosis hepática $(17,6 \%$ ), alrededor de los 40 años de edad; 2 èran alcohólicas rehabilitadas: en estas ultimas fue posible establecer que ingerian entre 2 y $3 \mathrm{~L}$ de vino al dia especialmente en los primeros meses del emb arazo.

La edad promedio de las madres durante el embarazo había sido 33 años y la mayoría de los recién nacidos $(\mathrm{RN})$, de término, sólo uno fue de pre-término, el peso promedio de los RN fue de $2.400 \mathrm{~g}$. La edad de los pacientes cuando se detectó la malfomación cardíaca fluctuaba entre 4 dias y 14 años y predominaban los varones $(11,17)$.

En cinco niños el defecto cardiaco era una comu nicación in terventricular $(29,2 \%)$. Se encontraron 4 casos con tetralogía de Fallot $(24,3 \%)$. En 2 existía drenaje venoso pulmonar anómalo (DVPA). En un caso cada uno: tronco arterioso, estenosis valvular pulmonar severa, estenosis aórtica subvalvular, comunicación interauricular (CIA), anillo vascular aórtico y ductus arterioso persistente (DAP).

En 3 pacientes la CIV era pequeña y se cerró espontáneamente en el primer año de vida, en otro caso, la CIV se asociaba con estenosis del infundíbulo pulmonar, en otro era de gran flujo, con hipertensión pulmonar.

Las tetralogías de Fallot eran extremas en 2 pacientes detectados en el período de RN y clásicas en otros dos. Los DVPA fueron en un caso de tipo total, infradiafragmático. $y$ se detectó en un RN y en el otro fue parcial, de las venas pulmonares izquierdas a la vena cava superior izquierda. El tronco arterioso fue de tipo 2 y se observó en un RN. La ClA era de tipo ostium secundum, siendo detectada en un pre-escolar. La estenosis valvular pulmonar severa fue descrita en un preescolar, la estenosis aórtica subvalvular también fue detectada en un escolar y era del tipo anillo fíbromuscular subaórtıco, sc asociaba con insuficiencia aórtica y mitral importantes. El anillo vascular aórtico correspondia á un caso de doble arco aórtico, ocurrió en un lactante $y$ el niño con ductus era un escolar.

Entre las anomalías extracardíacas asociadas at SAF se encontró polidactilia, situs inverso abdominal, atresia duodenal, criptorquidea, hipospadia con agenesia renal unilateral.
De los 17 pacientes con malformaciones cardiacas congénitas 5 fallecieron $(24 \%), 3$ en el periodo neonatal (tetralogía de Fallot extrema, tronco arterioso tipo 2, DVPA total infradiafragmático) y 2 en el postoperatorio (estenosis aórtica subvalvular y anillo vascular). Actualmente viven 12 pacientes, todos en capacidad funcional 1 (NYHA), 5 de ellos fueron operados con buenos resultados (CIV con hipertensión pulmonar, tetralogia de Fallot clásica, ductus, estenosis valvular pulmonar severa y CLA).

\section{COMENTARIO}

Las frecuencias de las diferentes $M C C$, en esta serie de pacientes con SAF, es semejante a la publicada por Sandor y col.12, y ambas fueron realizadas con semejantes métodos de investigación. En cambio en otra serie en que se realizaron. cate terismos cardíacos a todos los niños con $\mathrm{SAF}$ y soplos cardíacos durante el primer año đe vida predominaban los defectos septales inteauriculares $^{9}$.

La frecuencia de $\mathrm{MCC}$ en los pacientes con SAF resultó inferior en nuestra serie que en la Sandor ( 13 vs $41 \%)^{12}$. Estas diferencias pueden ser debidas a diferentes grados de expresión de los rasgos clínicos en el SAF relacionados con la modalidad de ingestión y la tolerancia individual. También puede influir la selección de la muestra, pues si se consideran sólo los $\mathrm{SAF}$ más severos, la frecuencia de cardiopatías asociadas será mayor. Asimismo otras diferencias dependen del método usado: si se practica solamente examen clínico. y son incluidos los soplos cardíacos banales, como se hizo en las primeras investigaciones de $\mathrm{SAF}$, la frecuencia de las cardiopatías es más alta ${ }^{9}$. También podría ser posible que el efecto teratogénico del alcohol fuese menor, debido a la cantidad inferior de alcohol consumido en el medio socioeconómico encuestado $(\bar{x} 90 \mathrm{cc}$ de alcohol puro en forma de vino, vs $180 \mathrm{cc}$ o más en bebidas de alto contenido de otras referencias) ${ }^{13}$. Además de las consideraciones anteriores, es necesario tener presente que el diagnóstico de MCC asociado con el SAF puede ser omitido, como consecuencia de muertes ocurridas en los primeros meses de vida, cierre espontáneo de defectos septales ${ }^{14}, y$ dificultades para obtener antecedentes de alcoholismo matemo.

No nos tue posible establecer relaciones entre el volumen de alcohol consumido en el embarazo y existencia de MCC por dificultades para obtener información precisa sobre los hábitos etílicos de las madres. La edad materna de la concepción de estos niños fue más bien tardía (TM 33 años) 
y la mortalidad de las madres por cirrosis hepatica, alta y precoz, en coincidencia con otras series en que $75 \%$ de las madres alcohólicas fallecieron 3 a 5 años después de concebir un hijo portador del síndrome 25 .

El pronóstico de los niños con MCC depende principalmente de la severidad de éstas, sin embargo es influido negativamente por el SAF puesto que al defecto cardiovascular se suman la deficiencia mental, que es constante, y el medio farniliar desfavorable 16,17,18.

Esta investigación subraya la existencia de causas de cardiopatía congénita potencialmente prevenibles. En efecto, el alcohol es uno de los principales teratógenos productores de cardiopatías junto con la Trimetadiona y la infección, durante el periodo gestional, por virus de rubéola, destacando así entre los pocos agentes detectables ${ }^{19}$. No está demas recordar que el alcohol es el teratógeno usado más frecuentemente $y$ libremente en el mundo.

Estamos convencidos que, evitando la ingestión de alcohol durante el embarazo, previniendo y controlando el alcoholismo, podrían disminuir, además de los problemas médicos de los pacientes con SAF, las consecuencias sociales de este hábito12,20,2].

\section{RESUMEN}

Entre 130 niños con sindrome alcohólico fetal (SAF) severo, controlados en las unidades de genética y cardiologia del hospital Guillemo Grant Benavente de Concepción, 17 tenían evidencia de malformaciones congénitas del corazón $(13 \%)$. Los defectos más frecuentes fueron el defecto septal interventricular $(29,2 \%)$ y la tetra. - logia de Fallot (24,3\%). Cinco de estos pacientes fallecieron (tres en el período neonatal y dos en el postoperatorio) y 12 tenian actualrnente buena capacidad funcional (cinco fueron sometidos a cirugía cardiovascular). Existe una asociación frecuente de cardiopatía congénita con SAF. El pronóstico de estos niños depende, no solamente de la severidad de la cardiopatía, sino también del déficit mental y los problemas sociales, relacionados con el hábito de ingerir alcohol: por lo que se destaca la importancia de su prevención.

\section{REFERENCIAS}

1. Rosser, H.L.; Weiner, L.: Strategies for prevention of Fetal Alcohol Effects. Obstet. Gynecol. 57: 1, 1981 .

2. Quazi, A.: Renal anomalies in Fetal Alcohol Syndrome. Pediatrics 63: 886, 1979.

3. Hovers, $W$., Majewski, $F$.: A nomalies of the Kidneys and Genitourinary tract in alcohol embriopathia. $J$. Urol. 124: 108, 1980.

4. Rasche, E.: New ophthalmic finding in Fetal Alcohol Syndrome. JAMA. 245: 108, 1981.

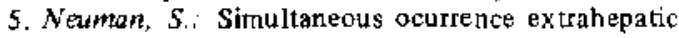
biliary atresia and Fetal Alcohol Syndrome, Am.J. Dis. Child. 133: 101, 1979.

6. Dunigan. T.H.; Werling, M.D.: Extrahepatic Biliary Atresia and renal anomalies in Fetal Alcohol Syndrome. Pediatrics 63: 886, 1979

7. Goldşein, $G$ : Neural tube defect and renal anomalies in a Child with F.A.S. Pediatries 93: 636. 1978.

8. Hanson, J.W.; Jones, K.L.; Smith, D.W.: Fetal Alcohol Syndrome. Experience with 41 patient. JAMA. 235: 1458, 1976.

9. Loser, H.: Majewski, F.: Type and frecuency of cardiac defects in embryofetal alcohol syndrome. Report of 16 cases. Br. Heart. J. 39: 1374, 1977.

10. Steeg, $C$; Woolf, $P$.: Cardiovascular malformations in the fetal alcohol Syndrome. Am. Heart. J, 98: $635,1979$.

11. Sandor, G.S.; Smith. D.W.: Cardiac malformations in the Fetal Alcohol Syndrome. J. Pediatr., 98: 771, 1981.

12. Clorren, S.X.: Recognition of Fetal Alcohol Syndrome. J.A.M.A. 45: 2436, 1981.

13. Joswb. S.: Fuchs, M.: Long Term follow-up of Three Siblings with Fetal Alcohol Syndtome. Alcoholism 5: 523, 1981.

14. Cockerham. J.T.i Martin, T.C.: Spontaneous closure of Secundum Atrial septal defect in Infants and Young Children. Am. J. Cardiol. 52: 1267, 1983.

15. Erb $L$, Andersen B.D.: The fetal alcohol syndrome: a review of the impact of chronic alcoholism in the development. Fetus Clin. Pediatr. 17: 645, 1978.

16. Mena $M$., Torres $C$.: Síndrome alcoholico fetal como causa de retardo mental prevenible. Rev. Chil. Pedjatr, 55: 114, 1984.

17. Schaywitz S.E., Cohen D.J.: Behaviour and learning difficulties in children of normal intelligence born of atcoholic mothers. J. Pediats. 96:978, 1980.

18. Poskint E.M., Hensey O.J.: Alcohol others drugs and the fetus. Der. Med. Child. Neurol. 24: 596, 1982 .

19. Nora J.J. Nora A.H.: The evolution of specific genetic and environmental counseling in congenital hearth diseases. Circulation 57: 205, 1978.

20. Larson $G$.: Prevention of fetal alcohol effects. Acta Obstet. Ginecol. Scand. 62: 171, 1983.

21. Plat P., Vedrine M.P.: Alcoolisation fóminine, grossesse et descendence. J. Gynecol. Obstet. Biol. Reprod. (París) 11: 969, 1982. 